Disclosure Information S. Schran: None. R. Datta: None. O. Persa: None. C. Aguilar: None. M. Thelen: None. J. Lehmann: None. M. Garcia-Marquez: None. K. Wennhold: None. A. Quaas: None. C. Bruns: None. C. Mauch: None. H. Löser: None. D. Stippel: None. H. Schlößer: None.

\section{P02.02 SINGLE-CELL RNA SEQUENCING OF NEUROBLASTOMA TUMORS REVEALS IMMUNOREGULATORY INTERACTIONS AS NOVEL TARGETS FOR IMMUNOTHERAPY}

J Wienke*, WM Kholosy, LL Visser, KM Keller, P Lijnzaad, T Margaritis, KPS Langenberg, RR De Krijger, FCP Holstege, JJ Molenaar. Princess Máxima Center for Pediatric Oncology, Utrecht, Netherlands

\subsection{6/jitc-2021-ITOC8.14}

Background Immunotherapy with CAR-T cells, as well as immune checkpoint blockade, show limited clinical efficacy in the pediatric solid cancer neuroblastoma, despite the success in various adult cancers. The lacking efficacy may be due to various immune evasion strategies employed by neuroblastoma tumors, leading to altered functionality of tumor-infiltrating immune cells. We aimed to provide a comprehensive overview of the composition and function of the neuroblastoma immune environment, as well as relevant immunoregulatory interactions $(=)$, to identify novel targets for immunotherapy. Materials and Methods 25 tumor samples from 20 patients (17 with high-risk disease, 6 with MYCN amplification), were collected during diagnostic biopsy pre-treatment $(n=10)$ or during resection surgery after induction chemotherapy $(n=15)$. Samples were enzymatically digested, single-cell FACS sorted and sequenced by Cel-Seq2 protocol.

Results Lymphoid cells in the TME consisted of $\alpha \beta-, \gamma \delta$-T cells, NK cells and B cells. Among $\alpha \beta-\mathrm{T}$ cells we identified $\mathrm{CD}^{+} \mathrm{T}$ cells, two functionally distinct clusters of $\mathrm{CD}^{+} \mathrm{T}$ cells, naive-like $\mathrm{T}$ cells and $\mathrm{FOXP}^{+}$regulatory $\mathrm{T}$ cells (Tregs). $\mathrm{CD}^{+} \mathrm{T}$ cells had reduced cytotoxic capacity compared to blood-derived $\mathrm{T}$ cells from a reference group. Tregs expressed high levels of PRDM1, LAYN and ICOS, suggesting an effector Treg profile, which is associated with increased inhibitory capacity. Although NK cells expressed the cytotoxic genes NKG7, KLRF1, GNLY, GZMB and PRF1, their expression was significantly lower than in blood-derived reference NK cells. Gene set enrichment analysis (GSEA) confirmed a reduced cytotoxic capacity of tumoral NK cells, which correlated with a decreased expression of activating receptors $(\mathrm{r}=0.41$, $\mathrm{p}<0.001)$ and increased TGF $\beta$ signaling $(\mathrm{r}=-0.45, \mathrm{p}<0.001)$. In addition, NK cells highly expressed the heterodimeric receptor KLRC1:KLRD1, which can inhibit NK cell function through HLA-E binding. High HLA-E expression by endothelial, immune and mesenchymal cells confirmed its inhibitory activity in the TME. Within the myeloid compartment we identified various immunosuppressive populations, comprising a cluster of IL10 and VEGFA expressing macrophages, three clusters of M2 differentiated macrophages expressing MMP9 and LGALS3, and dendritic cells with intact antigen presenting capacity, but high expression of numerous genes encoding immunosuppressive molecules such as IDO1, LGALS1, LGALS2, CCL22 and NECTIN2. In MYCN amplified tumors, specifically, we observed even lower cytotoxic capacity of $\mathrm{CD}^{+} \mathrm{T}$ and NK cells. We identified increased TGFB1 expression and defective antigen presentation by myeloid and tumor cells as potential causes for reduced cytotoxicity in MYCN amplified tumors. To identify relevant targets for immunotherapy we constructed an unbiased interaction network, which revealed NECTIN1 $=C D 96$ and $M I F=C D 74$ as active immunoregulatory interactions between tumor and T/NK cells, and CD80/CD86=CTLA4, CLEC2D $=$ KLRB1, HLA-E $=$ KLRC1/ KLRC2, CD99=PILRA, LGALS9=HAVCR2, and NECTIN2 =TIGIT between myeloid and T/NK cells.

Conclusions Cytotoxic lymphocytes in the neuroblastoma TME show reduced cytotoxic capacity, likely due to highly immunosuppressive myeloid cells, Tregs and numerous immunoregulatory interactions, which may serve as novel targets for immunotherapy in neuroblastoma.

Disclosure Information J. Wienke: None. W.M. Kholosy: None. L.L. Visser: None. K.M. Keller: None. P. Lijnzaad: None. T. Margaritis: None. K.P.S. Langenberg: None. R.R. De Krijger: None. F.C.P. Holstege: None. J.J. Molenaar: None.

\section{P02.03 AUTOMATED CELL TYPE SPECIFIC PD-L1 QUANTIFICATION BY ARTIFICIAL INTELLIGENCE USING HIGH THROUGHPUT BLEACH \& STAIN 15-MARKER MULTIPLEX FLUORESCENCE IMMUNOHISTOCHEMISTRY IN HUMAN CANCERS}

NC Blessin*, E Bady, T Mandelkow, C Yang, J Raedler, R Simon, C Fraune, M Lennartz, S Minner, E Burandt, D Höflmayer, G Sauter, SA Weidemann. University Medical Center Hamburg-Eppendorf, Hamburg, Germany

\subsection{6/jitc-2021-ITOC8.15}

Background The quantification of PD-L1 (programmed cell death ligand 1) has been used to predict patient's survival, to characterize the tumor immune microenvironment, and to predict response to immune checkpoint therapies. However, a framework to assess the PD-L1 status with a high interobserver reproducibility on tumor cells and different types of immune cells has yet to be established.

Materials and Methods To study the impact of PD-L1 expression on the tumor immune microenvironment and patient outcome, a framework for fully automated PD-L1 quantification on tumor cells and immune cells was established and validated. Automated PD-L1 quantification was facilitated by incorporating three different deep learning steps for the analysis of more than 80 different neoplasms from more than 10 '000 tumor specimens using a bleach \& stain 15-marker multiplex fluorescence immunohistochemistry panel (i.e., PDL1, PD-1, CTLA-4, panCK, CD68, CD163, CD11c, iNOS, CD3, CD8, CD4, FOXP3, CD20, Ki67, CD31). Clinicopathological parameter were available for more than 30 tumor entities and overall survival data were available for 1517 breast cancer specimens.

Results Comparing the automated deep-learning based PD-L1 quantification with conventional brightfield PD-L1 data revealed a high concordance in tumor cells $(\mathrm{p}<0.0001)$ as well as immune cells $(p<0.0001)$ and an accuracy of the automated PD-L1 quantification ranging from $90 \%$ to 95.2\%. Across all tumor entities, the PD-L1 expression level was significantly higher in distinct macrophage/dendritic cell (DC) subsets (identified by CD68, CD163, CD11c, iNOS; $\mathrm{p}<000.1)$ and in macrophages/DCs located in the Stroma $(p<0.0001)$ as compared to intratumoral macrophages/DC subsets. Across all different tumor entities, the PD-L1 
expression was highly variable and distinct PD-L1 driven immune phenotypes were identified based on the PD-L1 intensity on both tumor and immune cells, the distance between non-exhausted T-cell subsets (i.e. PD-1 and CTLA-4 expression on $\mathrm{CD}^{+}{ }^{+} \mathrm{CD} 8{ }^{+}$cytotoxic $\mathrm{T}$-cells, $\mathrm{CD} 3{ }^{+} \mathrm{CD} 4^{+} \mathrm{T}-$ helper cells, $\mathrm{CD}^{+}{ }^{+} \mathrm{CD} 4{ }^{+} \mathrm{FOXP} 3^{+}$regulatory $\mathrm{T}$-cells) and tumor cells as well as macrophage/(DC) subtypes. In breast cancer, the PD-L1 fluorescence intensity on tumor cells showed a significantly higher predictive performance for overall survival with an area under receiver operating curves (AUC) of $0.72(\mathrm{p}<0.0001)$ than the percentage of PD-L1 ${ }^{+}$ tumor cells (AUC: 0.54). In PD-L1 positive as well as negative breast cancers a close spatial relationship between $\mathrm{T}$ - cell subsets $\left(\mathrm{CD} 3{ }^{+} \mathrm{CD} 4{ }^{ \pm} \mathrm{CD} 8{ }^{ \pm} \mathrm{FOXP} 3{ }^{ \pm} \mathrm{PD}-1^{ \pm} \mathrm{CTLA}-4^{ \pm}\right)$and Macrophage/DC subsets $\left(\mathrm{CD} 68^{ \pm} \mathrm{CD} 163^{ \pm} \mathrm{CD} 11 \mathrm{c}^{\left.{ }^{ \pm} \mathrm{iNOS}\right)}\right.$ was found prognostic relevant $(p<0.0001)$.

Conclusions In conclusion, multiplex immunofluorescence PD-L1 assessment provides cutoff-free/continuous PD-L1 data which are superior to the conventional percentage of $\mathrm{PD}-\mathrm{L} 1^{+}$tumor cells and of high prognostic relevance. The combined analysis of spatial PD-L1/PD-1 data and more than 20 different immune cell subtypes of the immune tumor microenvironment revealed distinct PD-L1 immune phenotypes.

Disclosure Information N.C. Blessin: None. E. Bady: None. T. Mandelkow: None. C. Yang: None. J. Raedler: None. R. Simon: None. C. Fraune: None. M. Lennartz: None. S. Minner: None. E. Burandt: None. D. Höflmayer: None. G. Sauter: None. S.A. Weidemann: None.

\section{P02.04 TISSUE-INFILTRATING TH9 CELLS IN HUMAN ENDOMETRIAL CANCER}

${ }^{1} \mathrm{H}$ Tong ${ }^{*}, 2,3,4 \mathrm{H}$ Feng, ${ }^{1} \mathrm{X}$ Wan. ${ }^{1}$ Shanghai First Maternity and Infant Hospital, Shanghai, China; ${ }^{2}$ Department of Liver Surgery, Renji Hospital, School of Medicine, Shanghai Jiao Tong University, Shanghai, China; ${ }^{3}$ Shanghai Institute of Transplantation, Shanghai, China; ${ }^{4}$ Alumni Network of Ludwig-Maximilians University of Munich, Shanghai, China

\subsection{6/jitc-2021-ITOC8.16}

Background Endometrial cancer (EC) is a hormone-related carcinoma with increased morbidity among female patients of all backgrounds. The immune microenvironment of EC is uncertain.

Materials and Methods 102 patients were recruited in the present study. 90 postoperative specimens from the patients were analyzed by immunohistochemistry. The leukocyte landscape of endometrial cancer was mapped using high-dimensional single-cell profiling (CyTOF) for 12 patients.

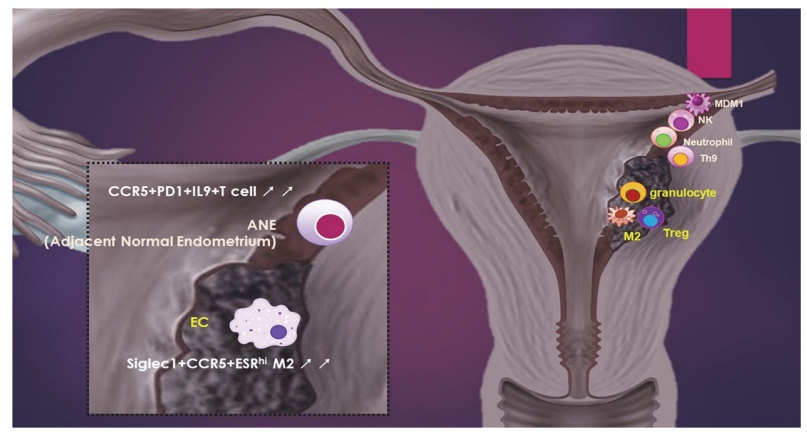

Abstract P02.04 Figure 1
Results NK cells, MDMs, and neutrophils were enriched in adjacent normal tissue. CCR5 + CD38+ PD1+Th9 cells were enriched in the invasive margin. Additionally, PD1+ESR $\mathrm{Eng}_{\text {eg }} \mathrm{T}$ cells and Siglec1+CCR5 +CD40+ESR ${ }^{\text {hi }}$ macrophage were infiltrated in the tumors.

Conclusions Immunological landscape of EC might shed light on new immunotherapuetic approach.

Disclosure Information H. Tong: None. H. Feng: None. X. Wan: None.

\section{P02.05 COMPREHENSIVE PROFILING OF TUMOR HETEROGENEITY AND ITS MICROENVIRONMENT IN ADVANCED NON-SMALL CELL LUNG CANCER AT SINGLE CELL RESOLUTION}

${ }^{1} \mathrm{Y}$ Cordts*, ${ }^{2} \mathrm{~F}$ Wu, ${ }^{1} \mathrm{~J}$ Fan, ${ }^{3} \mathrm{M}$ Odenthal, ${ }^{3} \mathrm{R}$ Buettner, ${ }^{2} \mathrm{C}$ Zhou. ${ }^{1}$ Singleron Biotechnologies, Cologne, Germany; ${ }^{2}$ Shanghai Pulmonary Hospital, Shanghai, China; ${ }^{3}$ University Hospital Cologne, Cologne, Germany

\subsection{6/jitc-2021-ITOC8.17}

Background Lung cancer is a highly heterogeneous disease. Cancer cells and cells within the tumor microenvironment together determine disease progression, as well as response to or escape from treatment.

Materials and Methods To map the cell type-specific transcriptome landscape of cancer cells and their tumor microenvironment in advanced non-small cell lung cancer (NSCLC), we analyzed 42 tissue biopsy samples from stage III/IV NSCLC patients by single cell RNA sequencing and presented the large scale, single cell resolution profiles of advanced NSCLCs.

Results In addition to cell types described in previous single cell studies of early stage lung cancer, we were able to identify new cell types such as follicular dendritic cells and $T$ helper 17 cells. Tumors from different patients display large heterogeneity in cellular composition, chromosomal structure, developmental trajectory, intercellular signaling network and phenotype dominance. Our study also revealed a correlation of tumor heterogeneity with tumor associated neutrophils, which might help to shed light on their function in NSCLC. Conclusions This study presented first-time the tumor heterogeneity and tumor microenvironment profile from late-stage, largely untreated NSCLC patients, and shed light on possible treatment regimes.

Disclosure Information Y. Cordts: A. Employment (full or part-time); Modest; Singleron Biotechnologies GmbH. F. Wu: None. J. Fan: A. Employment (full or part-time); Modest; Singleron Biotechnologies GmbH. M. Odenthal: None. R. Buettner: None. C. Zhou: None.

\section{P02.06 SEMI-AUTOMATED VALIDATION AND QUANTIFICATION OF CTLA-4 IN 90 DIFFERENT TUMOR ENTITIES USING MULTIPLE ANTIBODIES AND ARTIFICIAL INTELLIGENCE}

D Dum, TLC Henke, T Mandelkow, E Bady, R Simon, G Sauter, S Steuerer, W Wilczak, E Burandt, J Raedler, M Lennartz, NC Blessin*. University Medical Center HamburgEppendorf, Hamburg, Germany

\subsection{6/jitc-2021-ITOC8.18}

Background CTLA-4 is an inhibitory immune checkpoint receptor and a negative regulator of anti-tumor T-cell 